Población y Salud en Mesoamérica

Revista electrónica publicada por el Centro Centroamericano de Población, Universidad de Costa Rica, 2060 San José, Costa Rica

http://ccp.ucr.ac.cr

Población y Salud en Mesoamérica

Revista electrónica semestral, ISSN-1659-0201

Volumen 6, número 2, artículo 4

Enero - junio, 2009

Publicado 1 de enero, 2009

http://ccp.ucr.ac.cr/revista/

\title{
Patrones y flujos de la migración interna en la Gran Área Metropolitana de Costa Rica, en el período 1995- 2000
}

Wendy Molina-Varela 


\title{
Patrones y flujos de la migración interna en la Gran Área Metropolitana de Costa Rica, en el período 1995-2000
}

\section{Patterns and flows of internal migration at the Great Metropolitan Area of Costa Rica, in the period 1995-2000}

\author{
Wendy Molina-Varela ${ }^{1}$
}

\section{RESUMEN}

Los más recientes cambios en los patrones de migración interna en varios países de Latinoamérica, apuntan a los crecientes flujos de migración intrametropolitana desde los centros urbanos hacia la periferia, asociados a procesos de urbanización residencial de zonas rurales cercanas a la zona metropolitana. Este artículo analiza este fenómeno para Costa Rica, estimando los patrones de migración interna dentro del Gran Área Metropolitana (GAM), y tratando de determinar algunos factores de atracción y expulsión que posiblemente explican estos patrones, utilizando un modelo de Poisson para discriminar entre algunas variables seleccionadas en un modelo de regresión multivariable. Los resultados confirman algunas tendencias observadas para otras áreas metropolitanas de América Latina, y da algunas explicaciones al caso específico de la GAM.

Palabras clave: migración interna; migración urbana-urbana; Gran Área Metropolitana.

\begin{abstract}
The most recent patterns of internal migration in several Latin-American countries, pointed to the growing intrametropolitan flows from urban centers to peripheral areas, associated with urban residential processes in the rural areas next to metropolitan zone. This paper analyze this phenomenon in Costa Rica, estimating the patterns of internal migration within the Great Metropolitan Area (Gran Area Metropolitana - GAM), and trying to determine some push and pull factors that possibly explain these patterns, using a Poisson model to discriminate between a selected variables on a multivariate regression. The results confirm some tendencies observed in other metropolitan areas of Latin America, and give some explanations to the specific case of GAM.
\end{abstract}

Keywords: internal migration; urban-urban migration; Great Metropolitan Area (Gran Area Metropolitana- GAM).

Recibido: 29 jul. 2008

Aprobado: 9 oct. 2008

\footnotetext{
${ }^{1}$ Licenciada en Sociología. Funcionaria del Equipo Técnico de la Entidad Gestora del Proyecto Planificación Regional y Urbana en la Gran Área Metropolitana (PRUGAM), a cargo de la coordinación del Sistema Social del Plan PRUGAM 2008-2030. Costa Rica. molina29@ hotmail.com
} 


\section{INTRODUCCIÓN}

Un cambio significativo en los patrones de migración interna de algunos países latinoamericanos se observó, al menos de forma más clara, a partir de la ronda de censos del año 2000. Del examen de los microdatos censales de Bolivia, Brasil, Chile, Costa Rica, Guatemala y México, como parte del Proyecto BID/CEPAL "Migración y desarrollo: el caso de América Latina", se identificaron principalmente dos cambios importantes: la reversión del atractivo migratorio de las ciudades y la reestructuración interna de las metrópolis. Estos cambios se asocian a la mayor movilidad por migración intrametropolitana y sus implicaciones en materia de localización de los diferentes estratos sociales, actividades e infraestructura, como expresión del fenómeno de la segregación residencial (Rodríguez, 2007:02).

El patrón no fue idéntico para las seis metrópolis analizadas, operó como factor de distinción la cuantía demográfica de las ciudades, y de esta forma, las más pobladas (Rio de Janeiro, São Paulo y Santiago) tenían saldo neto negativo, mientras que las de menor tamaño demográfico, con la excepción de San José, registraban saldo positivo. No obstante, la observación de los datos permite identificar al menos cinco características en los patrones migratorios de todos los países analizados: coexisten o coinciden ciudades dentro de la misma metrópoli, como expulsoras y atractivas; las ciudades más pobladas tienen más probabilidad de ser expulsoras; una tendencia desconcentradora reduce el atractivo de las grandes ciudades y aumenta el de ciudades intermedias; se descarta la contraurbanización, es decir, los desplazamientos hacia zonas rurales; y finalmente, en algunos países -como Costa Rica- se observa un movimiento de "desconcentración concentrada", es decir, de migración "cercana" entre ciudades del mismo aglomerado metropolitano (Rodríguez, 2007: 05).

En relación con posibles factores de atracción y expulsión de población, lo hallado señala que estas recientes tendencias de migración interna están fomentadas por diferentes procesos de urbanización residencial, como asentamientos informales, proyectos gubernamentales de vivienda y barrios de estratos altos en zonas históricamente pobres o semirurales. Esta forma de urbanización se caracterizan por ocupar el territorio de forma dispersa y expansiva, configurando una gran periferia urbana de baja densidad (CELADE, 2006).

Esta extensión del uso del suelo residencial, se ha asociado con los procesos de reforma del Estado y desregulación económica, a partir de los cuales, progresivamente se ha producido un mayor déficit habitacional, un crecimiento urbano con una muy débil planificación y legislación urbana, y un peso creciente de los actores privados, muchos de ellos de capital extranjero, en la producción del entorno construido. De ahí la proliferación de asentamientos informales, la expansión desordenada de vivienda de interés social y el desarrollo de proyectos habitacionales del tipo condominio, barrio cerrado y complejo de oficinas, cuya tendencia es hacia la provisión de infraestructura y servicios urbanos otrora provistos por el Estado, los que en algunos casos pueden incluir hasta el equipamiento y servicios de salud y educación (Marín, 2006: 709).

En Costa Rica, en los últimos veinte años prácticamente no se ha producido investigación en materia de migración interna, debido en parte al tiempo que transcurrió sin realizarse el Censo Nacional de Población entre 1984 y 2000, lo que privó de datos a los investigadores especializados, y de otra parte debido al estudio prioritario de la inmigración internacional, 
principalmente de nicaragüenses, a partir de la década de los noventa (Barquero citado por CELADE, 2008).

A la luz de los resultados del Censo del 2000, se determinó que la provincia más expulsora fue Guanacaste, y la más atractora Heredia, seguida por Cartago y Alajuela (Chacón y otras, 2003). A propósito de esos resultados, se resaltó la importancia de las ciudades como atractoras de población y se lo atribuyó a la búsqueda de mejores condiciones de vida que se encuentran en centros urbanos con redes de servicios y equipamiento de salud y educación, a muy cortas distancias (Barquero, 2001). También se identificó la importante pérdida de población de los distritos que componen el casco central de San José, y se lo atribuyó a la progresiva reducción de la cantidad de hogares establecidos en la ciudad (Rosero, 2002).

Sin embargo, no se registran estudios que apuntaran hacia la identificación de los patrones de migración interna, hallados para otras metrópolis de Latinoamérica, ni que centren la atención del análisis en el conglomerado metropolitano, como se hace en el estudio de CEPAL mencionado anteriormente.

Con respecto a la relación entre la migración intrametropolitana y la extensión del suelo de uso residencial, que en otras metrópolis se ha asociado con el papel del Estado en la gestión del territorio, el panorama en Costa Rica apunta al desarrollo de estos mismos procesos en la Gran Área Metropolitana (GAM). Se ha determinado que la conformación de una amplia periferia urbana asociada con importantes desplazamientos de población, inició en la segunda mitad de la década de los años 80, gracias a las políticas de vivienda de la época: una serie de directrices permitieron la urbanización residencial fuera del anillo de contención urbana de la GAM, en la zona especial de protección agrícola y ambiental, e incorporaron a las entidades financieras y compañías constructoras privadas al desarrollo habitacional (Mora, 2003: 58).

Examinando la evolución del parque habitacional del período 2000-2006, se encuentra que las mayores y más numerosas inversiones en proyectos habitacionales recientes se han desarrollado en la GAM, por ejemplo, en el año 2005, 600000 de las más de un millón de viviendas del parque habitacional nacional, se ubicaban en la GAM, y el 85\% del PIB de la construcción en la rama de vivienda, se produjo en la GAM (Proyecto PRUGAM, 2007: 199).

En ese sentido, principalmente el sector financiero ha sido el agente económico que ha hecho posible el crecimiento del sector vivienda, el sector privado, en particular, financia principalmente a hogares de altos ingresos y en viviendas de tipo apartamento. Esto es el resultado de un mercado inmobiliario que se ha estructurado según los ingresos de los hogares y la oferta disponible, lo cual también ha dado como resultado una construcción de viviendas segmentada entre cantones de altos, medios y bajos ingresos (Proyecto PRUGAM, 2007: 203).

Considerando todo lo anterior, en el presente artículo se propuso determinar y analizar los patrones y flujos de migración interna del principal conglomerado urbano del país, a saber, la GAM2, y a la vez, explorar los posibles factores de atracción y expulsión de población a nivel intercantonal dentro de la GAM.

\footnotetext{
${ }^{2}$ Definida y delimitada mediante el Decreto Ejecutivo No $125,90-\mathrm{P}$. Dicha área incluye a 31 de los 81 cantones de Costa Rica, ocupa el 4.5\% del territorio nacional, y albergaba al año 2000 al 51\% de la población del país.
} 
La importancia de realizar este análisis a nivel de este aglomerado metropolitano, radica en que si bien solamente el 11\% (359 599) de la población mayor de 5 años, migró internamente entre 1995 y 2000, el 45\% de ésta (163 569) lo hizo entre cantones de la misma GAM.

Así, con el fin de determinar si efectivamente la migración interna en la GAM de Costa Rica se transforma en el sentido de lo observado en otras ciudades latinoamericanas, para el presente artículo se planteó la necesidad de explorar y analizar los siguientes aspectos:

a) La dirección de los principales flujos migratorios entre cantones, con el propósito de constatar si predominan los del centro a la periferia urbana, y que no está ocurriendo un proceso de contraurbanización.

b) Las características socioeconómicas de los cantones que más atraen o expulsan población, es decir, los posibles factores de atracción y expulsión.

c) El carácter atractor o expulsor de las ciudades más pobladas, de las ciudades intermedias y de la zona rural de la GAM, con el fin de constatar si se presenta la coexistencia de las condiciones atractoras y expulsoras en una misma ciudad.

\section{MATERIALES Y MÉTODOS}

El principal objetivo de la investigación de base del presente artículo, fue el de analizar los flujos migratorios internos dentro de la GAM y los posibles factores expulsores y atractores presentes en sus cantones, según la información que se procesó con datos del Censo 2000 para el período 1995-2000.

Para este propósito se trabajó en dos etapas: la primera fue la de identificar los principales patrones migratorios intercantonales en la GAM a partir de las tasas de migración reciente, y la segunda, consistió en determinar los posibles factores que inciden en la atracción y expulsión de población dentro de la GAM.

La matriz de migración interna constituyó el insumo fundamental para calcular las tasas de migración y los flujos intercantonales. Esta se construyó con información del Censo del año 2000, a partir del cruce entre el cantón de empadronamiento y el cantón de residencia cinco años antes, disponibles en la base de datos en línea del Centro Centroamericano de Población (CCP) en http://censos.ccp.ucr.ac.cr/.

Esta matriz con la información de los 31 cantones, permitió la determinación de los flujos migratorios y las tasas de migración, al aplicarse en un modelo de Poisson, mediante el que se realizó una regresión multivariada, utilizando la tasa de migración de cada cantón como variable dependiente, todo ello en el programa Stata.

Las variables independientes se definieron como factores asociados a la calidad del hábitat y las condiciones de vida en los cantones. Todo esto considerando lo hallado para las metrópolis de otros países latinoamericanos, con respecto a la importancia de la urbanización residencial en el 
motivo de los desplazamientos, así como el vertiginoso crecimiento de la oferta habitacional en la GAM, tal y como se explicó en la primera parte.

La principal variable dependiente es la del déficit habitacional. Considerando la importancia de la urbanización residencial en el motivo de los desplazamientos, se consideró que un mayor déficit habitacional en un cantón podría significar una menor inmigración, así como una mayor tendencia a ser un cantón expulsor de población.

Asociada al déficit habitacional, la variable densidad de viviendas, permite relacionar las tasas de migración con una mayor o menor densidad de viviendas, lo que a su vez es reflejo del déficit o superávit del parque habitacional.

En el ámbito espacial de la migración, la variable ubicación del cantón permite relacionar las tasas de migración con la dirección de la misma, es decir, si esta se produce en el centro o hacia la periferia urbana. Tal y como se explicó anteriormente, lo hallado en otras metrópolis latinoamericanas señala un mayor contingente de población migrante que se desplaza hacia la periferia urbana desde los centro poblados. Asimismo, dado el antecedente de la expansión del suelo urbano de la GAM durante la década de los 80s, fuera del anillo de contención urbana, y con ello, el retroceso en los logros de la planificación urbana, esta variable permite conocer el comportamiento actual de la urbanización en relación con las áreas establecidas como de potencial urbano y las zonas establecidas como de protección y producción agrícola fuera del anillo de contención urbana.

Por otro lado, las variables Índice de Necesidades Insatisfechas e Índice de Seguridad Ciudadana permiten asociar la migración intercantonal a la calidad del hábitat de cada lugar de origen y destino de los desplazamientos.

Asimismo, la concentración de la PEA en el sector terciario es una varible "proxi" de mayores fuentes de empleo, ya que en la GAM el 70\% de la actividad económica y de la PEA se concentra en el Sector Terciario, de manera que un mayor porcentaje de PEA en los Servicios y el Comercio, estaría indicando una mayor oferta laboral en sus diferentes ramas de actividad.

El Cuadro 1 en anexo, muestra la definición de todas las variables e indicadores utilizados en el modelo.

\section{RESULTADOS}

El análisis de las tasas de migración de cada uno de los cantones de la GAM, muestra claramente dos patrones: existe un área de cantones en la que ocurre gran movilidad de población y un área en la que los desplazamientos tanto de entrada como de salida son muy escasos, tal y como se puede observar en el Cuadro 2 anexo.

De un lado, en el área central y periférica de la GAM, en un grupo de 16 cantones que se ubican entre San Rafael, Alajuelita, Flores y Curridabat, se concentran las mayores tasas tanto de emigración como de inmigración. De otro lado, en un grupo de 11 cantones que se ubican principalmente en zonas agrícolas de Alajuela y Cartago, se concentran las menores tasas tanto de 
emigración como de inmigración. Y un restante grupo de 4 cantones, en los que la movilidad de la población se ubica entre los dos extremos anteriores.

De acuerdo con lo anterior, en un área central y periférica de la GAM, se produjo la mayoría de la migración. Los cantones que corresponden a los principales centros urbanos en el sistema jerárquico de ciudades de la GAM, como por ejemplo Montes de Oca, Tibás, San José y Heredia, resultaron altamente expulsores y atractores de población, pero con bajo saldo neto migratorio y algunos de signo negativo. Los cantones que en su mayoría se encuentran en la periferia urbana o zona rural en transición, que corresponden a ciudades intermedias en el sistema jerárquico de ciudades de la GAM, como por ejemplo Alajuelita, San Rafael, Flores y San Pablo, resultaron altamente expulsores y atractores de población, pero con alto saldo neto migratorio.

Asimismo, en el área limítrofe de la GAM, la que anteriormente fue zona especial de protección, se produjo la menor migración. Los cantones que corresponden principalmente a zonas agrícolas, a excepción de San José que se encuentra en este grupo por su baja tasa de inmigración, como por ejemplo Paraíso, Alvarado, Poás y Aserrí, resultaron escasamente expulsores y atractores de población ${ }^{3}$.

En relación con los factores que determinan los flujos de migración observados, los resultados de la regresión de Poisson que aparecen en el Cuadro 3 anexo, muestran que las características socioeconómicas de los cantones actúan muy débilmente como factores de atracción y expulsión de población.

Las características del cantón que tienen que ver con el parque habitacional existente y la condición socioeconómica del cantón, no alteran las tasas de migración. La regresión muestra que una alta densidad de viviendas o un mayor porcentaje de hogares con al menos una Necesidad Básica Insatisfecha en determinado cantón, no aumenta el riesgo de emigrar, aunque disminuye el riesgo de inmigrar, pero la diferencia entre uno y otro efecto es mínima, de 0.1 y 0.3 respectivamente.

Otras variables, aunque también influyen mínimamente en las tasas de migración, tienen la particularidad de que disminuyen tanto el riesgo de emigrar como de inmigrar, tal es el caso de la ubicación del cantón y la seguridad ciudadana. Así, el que un cantón se ubique dentro del anillo de contención, o tenga un mejor nivel de seguridad ciudadana, disminuye el riesgo de emigrar y también de inmigrar, pero su influencia en las tasas va de 0.1 a 0.2.

Finalmente, las fuentes de empleo y el mercado inmobiliario actúan aumentando, aunque de nuevo mínimamente, las tasas de migración. Una mayor concentración de la PEA en el sector terciario, o un mayor déficit habitacional, aumentan tanto el riesgo de emigrar, como de inmigrar a determinado cantón. Pero en el caso de la variable déficit habitacional, es mayor el riesgo de inmigrar que de emigrar, con una diferencia de 0.21 .

\footnotetext{
${ }^{3}$ Ver mapa anexo.
} 


\section{DISCUSIÓN}

Al igual que se presenta en los patrones migratorios internos de otros países latinoamericanos, las tasas de migración de los cantones de la GAM muestran que la dirección de los flujos migratorios son del centro hacia la periferia urbana. Asimismo, los centros urbanos más poblados e importantes en el sistema jerárquico de ciudades son expulsores, mientras que la periferia urbana crece por inmigración.

Por su parte, las áreas rurales casi no participan en la migración interna de la GAM, ya que tanto la salida como la entrada de población es muy escasa. Además, debido a que los mayores flujos migratorios se desplazan del centro a la periferia urbana y no hacia estas áreas rurales, no se puede afirmar que se presente contraurbanización.

Las características socioeconómicas de los cantones actúan muy débilmente como fuerzas atractoras o expulsoras de población, según lo mostraron los resultados de la regresión, ya que el efecto de las variables independientes sobre las tasas de migración resultó mínimo. Sin embargo, es importante hacer notar que hay unas variables que más inciden en la migración, es decir, que aumentan tanto el riesgo de emigrar como de inmigrar, a diferencia de todas las restantes que disminuyen o simplemente no alteran el riesgo de migrar.

De manera que mientras algunas variables generan movilidad, otras no alteran el riesgo de migrar, entre ellas, algunas retienen población.

Las variables que influyen de forma positiva en la migración son: la concentración de la PEA en el sector terciario y el déficit habitacional, variables más ligadas a la dinámica económica que al lugar que ocupa el cantón o a características socioeconómicas del mismo.

De acuerdo con las características de los cantones de alta y baja movilidad, así como con las variables más influyentes en la migración, es claro que son los cantones más dinámicos económicamente, con mayor desarrollo y oferta inmobiliaria y laboral, más densamente poblados y que se ubican en los centros urbanos, los que debido a éstas características atraen y expulsan población. Por el contrario, los cantones de baja migración, se caracterizan por un mercado inmobiliario y laboral menos activo, dada su vocación agrícola y su lento crecimiento del parque habitacional.

Estos resultados señalan una tendencia observada en las metrópolis de otros países latinoamericanos, en las que la urbanización residencial de las afueras de la ciudad, actúan como principal factor de movilización de población, tanto por la oferta privada de vivienda en condominio o barrio cerrado, como por la vivienda de interés social o el asentamiento informal, todo ello en un proceso de colonización de tierras otrora agrícolas, fomentado por políticas de liberalización del mercado inmobiliario.

En el caso de la GAM se confirma el desarrollo de esos mismos procesos, como las tomas de tierra para establecer asentamientos en precario y el desarrollo de numerosos proyectos de vivienda de interés social, durante la década de los años 80 , y más recientemente, la proliferación de condominios y barrios cerrados en zonas agrícolas de rápido proceso de urbanización como ocurre en algunas áreas de Escazú, Santa Ana y las faldas montañosas de Heredia. 
Las "nuevas" migraciones internas señalan la búsqueda de vivienda como principal factor de movilización de población, y no la búsqueda de empleo que orientaba los flujos desde las zonas de economía extractiva o estancada, hacia centros urbanos dinámicos integrados a la economía internacional, que se registró y explicó así durante la década de los años 70, a partir de las teorías clásicas en el estudio de las migraciones latinoamericanas.

Sin embargo, en los patrones actuales, es evidente la persistencia de polos de desarrollo en contraposición de áreas deprimidas, así como desigualdades regionales de productividad e ingreso, lo cual resalta la actualidad de los rasgos típicos de los estilos de desarrollo prevalecientes en América Latina, dado el origen y desarrollo histórico de su estructura económica, ahora complejizados en un contexto de globalización, con un cambio en el estilo de desarrollo que debilitó el papel del Estado y fortaleció la injerencia del sector privado y el capital internacional en la producción del espacio urbano y la gestión de la ciudad.

Finalmente, todos estos elementos señalan la importancia de desarrollar un cuerpo teórico para el estudio de las migraciones intrametropolitanas, que dé cuenta de las manifestaciones actuales de los problemas estructurales de "siempre", y que conduzca a explicar más claramente los nuevos patrones de migración interna en los países de Latinoamérica. De manera que se facilite la compresión de los procesos apuntados en este trabajo y que requieren un adecuado ordenamiento territorial y nuevas estrategias de planificación urbana. 


\section{BIBLIOGRAFÍA}

Barquero, J. (2001, agosto 23). Ciudades atraen más gente. La Nación, p. 8. http://www.nacion.com/ln_ee/2001/agosto/23/pais8.html (Consultado el 1 de julio 2008)

CELADE (2006). Migración interna muestra signos de transformación. Temas de población y desarrollo, Número 6, Santiago de Chile.

CELADE (2008) Migración y Desarrollo: el caso de América Latina. Informe compilado de las actividades de difusión: talleres nacionales y seminario internacional. Distribución limitada: Santiago de Chile.

http://www.eclac.cl/cgi-bin/getProd.asp?xml=/celade/noticias/noticias/5/32075/P32075. $\mathrm{xml} \& \mathrm{xsl}=/$ celade/tpl/p1f.xsl\&base=/celade/tpl/top-bottom.xsl (Consultado el 1 de julio 2008).

Chacón, Ramírez y Retana (2003). Movilidad intercantonal de la población en Costa Rica 19842000. Factores determinantes y consecuencias. Tesis para optar por el título de grado de Licenciatura en Sociología, Escuela de Antropología y Sociología, Universidad de Costa Rica.

Herrera, R. (2006). La perspectiva teórica en el estudio de las migraciones. Editorial Siglo XXI: Ciudad de México.

Mora. J. (2003). Análisis del crecimiento urbano de la Gran Área Metropolitana de Costa Rica. Período 1983-2000. Tesis para optar por el título de grado de Magíster en Geografía, Escuela de Geografía, Universidad de Costa Rica.

PRUGAM (2007). Estudio Económico de la GAM. Distribución limitada: San José.

PRUGAM (2007). Estudio de Aspectos Sociales de la GAM. Distribución limitada: San José.

Ríos, D. (2006). Cambios en la producción del espacio urbano residencial en la periferia de la ciudad metropolitana de Buenos Aires. Un estudio de caso en el municipio de Tigre. Estudios Demográficos y urbanos. Vol. 21 (63), p. 701-729.

Rodríguez, J. (2007) Dinámica demográfica y asuntos urbanos y metropolitanos en América Latina: ¿qué aporta el procesamiento de microdatos censales?. Distribución limitada: Santiago de Chile.

Rosero, L. (2002, mayo 13). San José, desierto urbano. Éxodo y envejecimiento de la capital. $L a$ Nación, p. 15. http://www.nacion.com/ln_ee/2002/mayo/13/opinion3.html (Consultado el 1 de julio 2008) 


\section{ANEXOS}

Cuadro 1. Definición de variables del modelo de regresión.

\begin{tabular}{|c|c|c|c|}
\hline $\begin{array}{l}\text { Característica del } \\
\text { cantón }\end{array}$ & Variable & Indicador & Fuente \\
\hline $\begin{array}{l}\text { Mercado } \\
\text { inmobiliario }\end{array}$ & Déficit habitacional & $\begin{array}{l}\text { Porcentaje de } \\
\text { hogares sin } \\
\text { vivienda por } \\
\text { cantón }\end{array}$ & Censo 2000 \\
\hline Parque habitacional & Densidad de viviendas & Viviendas/Km2 & $\begin{array}{l}\text { Censo 2000 } \\
\text { y datos } \\
\text { PRUGAM }\end{array}$ \\
\hline $\begin{array}{l}\text { Condición } \\
\text { socioeconómica del } \\
\text { cantón }\end{array}$ & $\begin{array}{l}\text { Índice de Necesidades } \\
\text { Básicas Insatisfechas } \\
\text { (NBI) }\end{array}$ & $\begin{array}{l}\text { Porcentaje de } \\
\text { hogares con al } \\
\text { menos una } \\
\text { Necesidad } \\
\text { Básica } \\
\text { Insatisfecha por } \\
\text { cantón }\end{array}$ & Censo 2000 \\
\hline Fuentes de empleo & $\begin{array}{l}\text { Concentración de la } \\
\text { PEA en el sector } \\
\text { terciario. }\end{array}$ & $\begin{array}{l}\text { Porcentaje de la } \\
\text { PEA empleada } \\
\text { en el Sector } \\
\text { Terciario por } \\
\text { cantón }\end{array}$ & Censo 2000 \\
\hline Seguridad ciudadana & $\begin{array}{l}\text { Índice de Seguridad } \\
\text { Ciudadana }\end{array}$ & $\begin{array}{l}\text { Puntuación en } \\
\text { el ISC por } \\
\text { cantón }\end{array}$ & $\begin{array}{l}\text { ISC. PNUD } \\
2003\end{array}$ \\
\hline $\begin{array}{l}\text { Ubicación del } \\
\text { cantón }\end{array}$ & $\begin{array}{l}\text { Ubicación central o } \\
\text { periférica del cantón } \\
\text { (dentro o fuera del } \\
\text { anillo de contención } \\
\text { urbana). }\end{array}$ & Centro/periferia & $\begin{array}{l}\text { Datos } \\
\text { PRUGAM }\end{array}$ \\
\hline
\end{tabular}


Cuadro 2. Mayores y menores Tasas de Emigración e Inmigración. GAM, 1995-2000.

\begin{tabular}{|c|c|c|c|c|c|c|c|}
\hline \multicolumn{2}{|c|}{ Cantones de alta movilidad } & \multicolumn{3}{c|}{ Cantones de baja movilidad } \\
Tasas de Migración & \multicolumn{2}{c|}{ Inmigración } & \multicolumn{3}{c|}{ Emigración } & Inmigración \\
\hline Emigración & 32.29 & Moravia & 33.97 & Atenas & 5.21 & Paraíso & 8.94 \\
\hline M. de Oca & 29.73 & Coronado & 31.78 & Alajuela & 6.10 & Atenas & 9.05 \\
\hline Tibás & 28.43 & Flores & 31.15 & Poás & 8.17 & Alajuela & 9.65 \\
\hline San José & 24.84 & San Pablo & 30.21 & Oreamuno & 8.21 & Alvarado & 10.40 \\
\hline Goicoechea & 24.39 & Curridabat & 29.44 & Paraíso & 8.43 & Poás & 11.00 \\
\hline Moravia & 22.98 & S. Rafael & 27.65 & Mora & 8.53 & Cartago & 11.98 \\
\hline Heredia & 22.69 & M. de Oca & 26.38 & Alvarado & 8.96 & San José & 12.60 \\
\hline Curridabat & 21.76 & Alajuelita & 25.51 & Cartago & 10.59 & Mora & 14.29 \\
\hline Escazú & 21.22 & S.Domingo & 25.11 & El Guarco & 12.23 & Oreamuno & 15.87 \\
\hline San Pablo & 19.90 & San Isidro & 24.86 & Aserrí & 12.69 & El Guarco & 16.14 \\
\hline Flores & & & & & \\
\hline
\end{tabular}

Cuadro 3. Riesgos relativos de migrar entre cantones. GAM, 1995-2000.

\begin{tabular}{|c|c|c|c|c|}
\hline & Migrantes & IRR & $95 \%$ Conf. & Interval \\
\hline \multirow[t]{6}{*}{ Cantón de origen } & $\operatorname{viv} / \mathrm{Km}^{2}$ & 1.0001 & 1.0000 & 1.0001 \\
\hline & INBI & 1.0059 & 1.0045 & 1.0074 \\
\hline & ISC & 0.9972 & 0.9967 & 0.9967 \\
\hline & $\%$ PEA sector $3^{\mathrm{a}}$ & 1.0335 & 1.0324 & 1.0346 \\
\hline & Centro urbano & 0.8103 & 0.7955 & 0.8254 \\
\hline & Déficit habitacional & 1.2898 & 1.2680 & 1.3121 \\
\hline \multirow[t]{8}{*}{ Cantón de destino } & Población & 1.0000 & 1.0000 & 1.0000 \\
\hline & viv $/ \mathrm{Km}^{2}$ & 0.9996 & 0.9996 & 0.9996 \\
\hline & INBI & 0.9757 & 0.9743 & 0.9771 \\
\hline & ISC & 0.9956 & 0.9949 & 0.9963 \\
\hline & $\%$ PEA sector $3^{\mathrm{a}}$ & 1.0372 & 1.0362 & 1.0382 \\
\hline & Centro urbano & 0.9700 & 0.9540 & 0.9862 \\
\hline & Déficit habitacional & 1.4970 & 1.4742 & 1.5201 \\
\hline & Población Origen (exposure) & & & \\
\hline
\end{tabular}




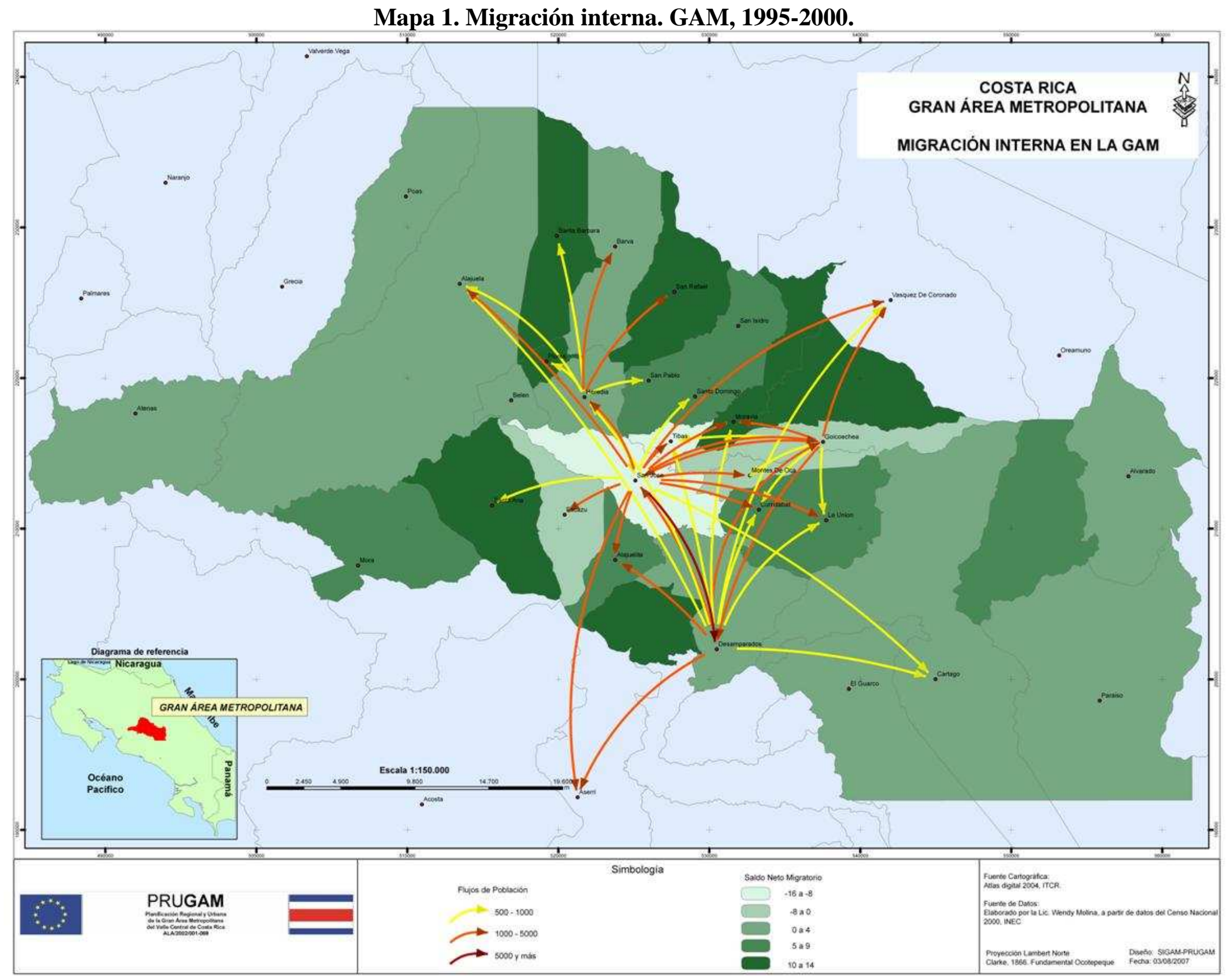

